\title{
Neuroimaging Indices Of Vascular Cognitive Impairment After Stroke In a Sample Of Egyptian Patients
}

\author{
Ahmed Essmat, ${ }^{1}$ MD, Sayed El-Zayat, ${ }^{1}$ PhD, Mahmoud I. Elshamy, ${ }^{2}$ PhD, Mostafa S. Badawy, ${ }^{1}$ Msc.
}

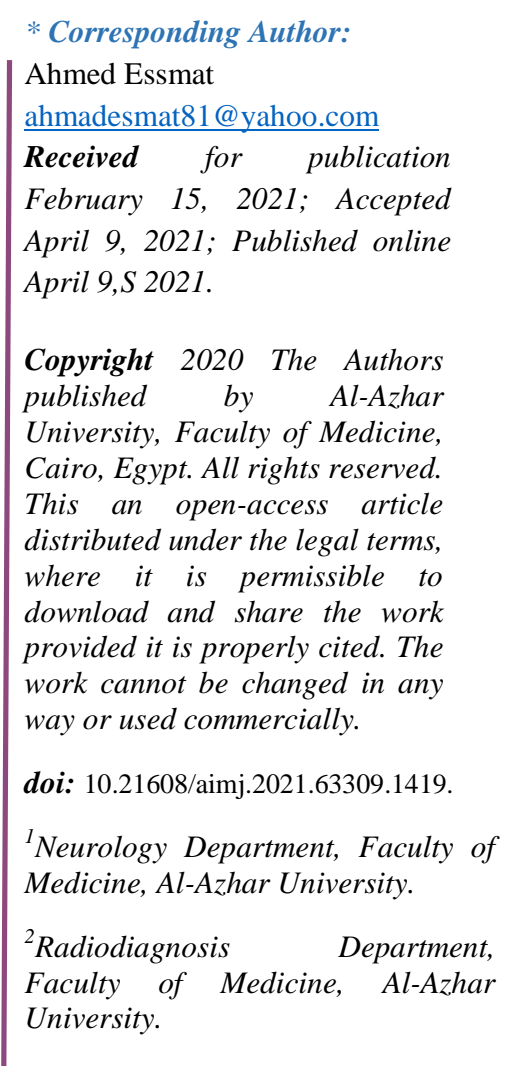

\begin{abstract}
Background: The second prevalent cause of death globally is cerebrovascular stroke. It's responsible for a great extent of disabilities than any other condition. A large share of this disability is related to poststroke neurocognitive impairment.

Aim of the study: This study aimed to assess neuroimaging indices related to cognitive dysfunction in a sample of Egyptian stroke patients. Patients and Methods: This study included 70 patients diagnosed with ischemic or hemorrhagic stroke after 3 months from the onset. Cognitive functions were assessed using the Montreal Cognitive Assessment test (MoCA).

Results: This study included 70 stroke patients. They comprised 29 men and 41 women with an age of $52.9 \pm 10.4$ years. According to MoCA score, cognitive impairment was detected in 22 patients (31.4\%) while the remainder 48 patients had normal cognitive function. Patients with cognitive impairment are significantly older $(59.6 \pm 6.2$ versus $49.9 \pm$ 10.6 years; $\mathrm{p}<0.001$ ) and have significantly higher frequency of atherosclerotic disease (64\% versus $36 \%, \mathrm{p}<0.001$ ). It was found that patients with cognitive impairment show significantly higher frequency of left hemisphere strokes ( $68 \%$ versus $42 \%$; $p=0.039$ ), both cortical and deep strokes (59 \% versus $18 \%$ and $23 \%$; $\mathrm{p}=0.042$ ). It was also demonstrated that patients with cognitive impairment had significantly larger stroke lesions when compared with patients with normal cognition $(12.8 \pm 1.9$ versus $3.9 \pm 0.9 \mathrm{~cm} ; \mathrm{p}<0.001)$.

Conclusion: Cognitive impairment is prevalent in stroke patients. Risk factors included older age, left hemispheres strokes, cortical strokes and larger stroke lesions.
\end{abstract}

Keywords: Stroke; Cognitive impairment; Neuroimaging.

\section{INTRODUCTION}

The second major reason of death worldwide is cerebrovascular stroke. It's responsible for a great extent of disabilities than any other condition ${ }^{1}$. A large share of this disability is related to poststroke neurocognitive disorders as cognitive impairment ${ }^{2}$. The burden of stroke is likely to increase over the next few year on society, due to occurring demographic changes as aging of the population ${ }^{3}$.

In the success of most everyday tasks, cognitive abilities play a vital role, so assessing them is an essential part of stroke rehabilitation and treatment. One of the most serious problems affecting stroke survivors is cognitive dysfunction. Through their recovery, approximately $80 \%$ of stroke patients undergo a degree of cognitive disability ${ }^{4}$. Greater risk of impairment, poorer quality of life, symptoms of depression, and mortality are linked to it ${ }^{5}$.

Cognitive impairment prevalence varies according to evaluation techniques, descriptions, or characteristics of the sample ${ }^{6}$. The use of a suitable evidence-based method for early detection of cognitive impairment lessen the risk of cognitive impairment post stroke ${ }^{7}$.

Vascular cognitive impairment (VCI) is a condition that encompasses all cognitive disabilities due to different types of cerebral vascular disease and related risk factors. It typically occurs to higher brain functions, particularly memory and executive functions ${ }^{8}$.

Neuroimaging is crucial for detecting and treating vascular cognitive dysfunction, as it gives vital information on the neuroanatomical substrate of the 
disease and the diagnosis of white and gray matter with ischaemic or hemorrhagic injury ${ }^{9}$.

This study aimed to evaluate the vascular cognitive Impairment in relation to neuroimaging indices as regard site right or left hemisphere, strategic location, and size of stroke in a sample of Egyptian stroke patients.

\section{PATIENTS AND METHODS}

\section{Ethical considerations:}

-The study protocol was accepted by the ethics committee of Al-Azhar University's faculty of medicine.

-An informed written consent was taken from all the participants in the study or their relative.

Patients: This descriptive study was conducted at neurology department, Sohag Teaching Hospital, Sohag, Egypt. The study included 70 patients diagnosed with ischemic or hemorrhagic stroke after 3 months from the onset, during the period from May 2019 to April 2020.

\section{Inclusion criteria:}

Patient diagnosed ischemic or hemorrhagic stroke after 3 months from the event and their age not above 65 years old with no history of cognitive impairment before stroke ${ }^{10}$.

\section{Exclusion criteria:}

- Patients with transient ischemic attack.

- Aphasia patients (on the language component of the National Institute of Health Stroke Scale (NIHSS) score $\geq 1$.

- Patients with cognitive impairment other than vascular insult (e.g., Parkinsonism ,...).

- Mental subnormality.

- Psychiatric diseases such as (depression).

\section{All of the participants were be subjected to the} following:

-Detailed medical and neurological history.

-Detailed medical and neurological examination.

-Laboratory investigation as Complete Blood Count, liver function tests, Lipogram, Renal function tests, Random blood sugar.

-Radiological investigations as Magnetic resonance imaging or Computed topography.

-Montreal Cognitive Assessment test (MoCA) I \& II, adding extra point to patients with $\leq 12$ years of education $^{11 .}$
-Hamilton depression rating scale.

\section{Statistical analysis:}

Data from this study were reported as percentage and number or mean \pm standard deviation (SD). Categorical data were compared using Fisher's exact test while numerical data were compared by $\mathrm{T}$ test. All statistical procedures were processed by SPSS, 26 and $\mathrm{p}$ value less than 0.05 was considered statistically significant.

\section{RESULTS}

This study included 70 patients diagnosed stroke. They comprised 29 men and 41 women with an age of $52.9 \pm 10.4$ years. According to MoCA score, cognitive impairment was detected in 22 patients (31.4\%) while the remainder 48 patients had normal cognitive function. Comparison between cognitive impairment patients and patients with normal cognition regarding the clinical characteristics revealed that those with cognitive impairment are significantly older $(59.6 \pm 6.2$ versus $49.9 \pm 10.6$ years; have significantly higher frequency of atherosclerotic disease (64 \% versus $36 \%, \mathrm{p}<0.001$ ), have significantly higher frequency of smoking $(54.6 \%$ versus $25 \%, P$ value $=0.016)$, have significantly higher frequency of hyperlipidemia $(68 \%$ versus $32 \%, \mathrm{P}$ value $=0.006)$, have significantly higher frequency of multiple risk factors ( $86 \%$ versus $14 \%, \mathrm{p}<0.001$ ), and have significantly higher frequency of lower level of education $(77 \%$ versus $23 \%, P$ value $=0.042)($ Table- 1$)$.

Regarding the neuroimaging findings, patients with cognitive impairment showed significantly higher frequency of left hemisphere strokes (68\% versus 42 $\% ; \mathrm{p}=0.039$ ), both (cortical-subcortical) stroke (59 \% versus $18.6 \%$ and $23 \%$; $=0.046)$. Patients with cognitive impairment had significantly larger stroke lesions when compared with patients with normal cognition $(12.8 \pm 1.9$ versus $3.9 \pm 0.9 \mathrm{~cm} ; \mathrm{p}<0.001)$. (Table-2).

\section{DISCUSSION}

\section{Discussion}

Based on Montreal Cognitive Assessment in this report there was 22 (31.4 \%) patients with cognitive impairment. Earlier recorded result of cognitive impairment rates is consistent with this study, which based on follow-up period and subtype of stroke, can range from around 30 percent to 74 percent ${ }^{12}$.

In this study, cognitive dysfuction patients were significantly older in line with previous reports. Kalaria et al. ${ }^{13}$ found that older age is a strong predictor for cognitive dysfunction after stroke. Also, Renjen et al. ${ }^{14}$. found that cognitive impairment frequency was significantly elevated with increasing age.

This study revealed a highly statistically significance between atherosclerosis and cognitive impairment with ( $\mathrm{P}$ value $<0.001$ ). 
Studies by Wang et al. ${ }^{15}$, Zhong et al. ${ }^{8}$, and Arntzen et al. ${ }^{16}$ showed that there was statistically significance between atherosclerosis and cognitive impairment.

This study revealed a statistically significance between smoking and cognitive impairment with ( $\mathrm{P}$ value $<0.016$ ).

In the study by Filip et al. ${ }^{17}$ there was worse performance of memory and cognitive function after adjustment for age, educational attainment, which support this study result. Rusanen et al. ${ }^{18}$ found that middle age heavy smoking doubled the possibility of older age cognitive impairment.

On the other hand a study by Sabia et al. ${ }^{19}$ showed that smoking and cognitive impairment have not been linked, but the underlying causes remain uncertain.

In this study, there was a statistically significance between hyperlipidemia, and cognitive impairment (P value <0.046).
In the studies by Reijmer et al. ${ }^{20}$, and Akinyemi et al. 21 there was statistically significant difference between hyperlipidemia and cognitive impairment function.

On the other hand, Yannick et al. ${ }^{22}$ study showed that post-stroke cognitive dysfunction incidence was lower in patients with hyperlipedemia than in patients without hyperlipedemia.

In this study, as regard to predisposing factors , there was no statistically significance between hypertension, diabetes, and cognitive impairment.

A study by Renjen et al. ${ }^{14}$ showed that there was no cognitive disability correlation was found with the degree of blood sugar levels regulation. In the study of Yannick et al. ${ }^{22}$ there were no relation between post stroke cognitive impairment and either ischemic heart disease or hypertension.

\begin{tabular}{|l|l|l|l|l|}
\hline & $\begin{array}{l}\text { All patients } \\
\mathrm{N}=70\end{array}$ & $\begin{array}{l}\text { Cognitive impairment } \\
\mathrm{n}=22\end{array}$ & $\begin{array}{l}\text { Normal cognition } \\
\mathrm{n}=48\end{array}$ & P value \\
\hline $\begin{array}{l}\text { Age (years) mean } \pm \\
\text { SD }\end{array}$ & $52.9 \pm 10.4$ & $59.6 \pm 6.2$ & $49.9 \pm 10.6$ & $<0.001^{*}$ \\
\hline Male/female n & $29 / 41$ & $9 / 13$ & $20 / 28$ & 0.95 \\
\hline Comorbidities n (\%) & $24(34.3)$ & $12(54.6)$ & $12(25.0)$ & $0.016^{*}$ \\
\hline Smoking & $25(48)$ & $14(64)$ & $11(36)$ & $<0.001^{*}$ \\
\hline Atherosclerosis & $32(45.7)$ & $11(50.0)$ & $21(43.8)$ & 0.63 \\
\hline Hypertension & $23(32.9)$ & $8(36.4)$ & $15(31.3)$ & 0.67 \\
\hline Diabetes mellitus & $47(67)$ & $15(68)$ & $32(66)$ & $0.046^{*}$ \\
\hline Dyslipidemia & & & & 0.83 \\
\hline One risk factor & $17(24)$ & $3(18)$ & $14(29)$ & - \\
\hline Two risk factors & $26(37)$ & $8(36)$ & $6(12.5)$ & - \\
\hline Three risk factors & $10(14)$ & $4(18)$ & $5(10)$ & - \\
\hline Four risk factors & $13(19)$ & $8(36)$ & $30(62.5)$ & $<0.001^{*}$ \\
\hline Multiple risk factors & $49(70)$ & $19(86)$ & $27(56)$ & $0.042^{*}$ \\
\hline Education $>12$ years & $32(46)$ & $5(23)$ & $21(44)$ & \\
\hline Education $\leq 12$ years & $38(54)$ & $17(77)$ & & \\
\hline
\end{tabular}

Table 1: Clinical characteristics in the studied patients (* significant results).

\begin{tabular}{|c|c|c|c|c|}
\hline & $\begin{array}{l}\text { All } \\
\text { patients } \\
\mathrm{N}=70\end{array}$ & $\begin{array}{l}\text { Cognitive } \\
\text { impairment } \\
n=22\end{array}$ & $\begin{array}{l}\text { Normal cognition } \\
n=48\end{array}$ & $\mathrm{P}$ value \\
\hline \multicolumn{5}{|l|}{ Type n (\%) } \\
\hline Hemorrhage & $18(25.7)$ & $6(27.3)$ & $12(25.0)$ & \multirow{2}{*}{0.84} \\
\hline Infarction & 52 (74.3) & $16(72.7)$ & $36(75.0)$ & \\
\hline \multicolumn{5}{|l|}{ Location n (\%) } \\
\hline Right hemisphere & $32(45.7)$ & $7(31.8)$ & $28(52)$ & \multirow{2}{*}{$0.039 *$} \\
\hline Left hemisphere & 38 (54.3) & $15(68.2)$ & $23(48)$ & \\
\hline \multicolumn{5}{|l|}{ Site n (\%) } \\
\hline Cortical-Subcortical & 23 (32.9) & $13(59)$ & $10(21)$ & \multirow{3}{*}{$0.042^{*}$} \\
\hline Cortical & $13(18.6)$ & $4(23)$ & $9(19)$ & \\
\hline Subcortical & $34(48.6)$ & $5(18)$ & $29(60)$ & \\
\hline Size $(\mathrm{cm})$ mean \pm SD & $7.8 \pm 1.3$ & $12.8 \pm 1.9$ & $3.9 \pm 0.9$ & $<0.001 *$ \\
\hline
\end{tabular}

Table 2: Stroke lesion characteristics in the studied patients (* significant results).

On the other hand a study by Kalaria et al. ${ }^{13}$ showed that hypertention is linked with an increased incidence of stroke and cognitive impairment associated with stroke extension. The period of 
hypertension is a significant determinant of cognitive impairment after stroke, in addition to the severity of high blood pressure.

In this study, there was a statistically significance between patients with multiple risk factors, and cognitive impairment (P value $<0.001$ ).

A study by Kalaria et al. ${ }^{13}$ mentioned that presence of neurodegenerative changes or multible vascular risk factors render the brain more vulnerable to cognitive decline. Chen et al. ${ }^{23}$ mentioned that the existence of midlife multible risk factors (i.e., hyperlipidemia, hypertention , and cerebrovascular disease) is linked to an increased risk of vascular cognitive impairment.

In this study, there was a statistically significance between lower education, and cognitive impairment ( $\mathrm{P}$ value $<0.042$ ).

This in agreement with Tu Q et al. ${ }^{24}$ which showed that low education had significant effects on cognition post stroke.

Interestingly, the present study found no relation between stroke type and cognitive impairment in agreement with the study of Yang et al. ${ }^{25}$. On the other hand, a study by Renjen et al. ${ }^{14}$ showed that in comparison to patients with ischemic strokes, more patients with hemorrhagic strokes have cognitive dysfunction.

In this study, patients with cognitive impairment had statistically significance with left hemispheric stroke. Likewise, Renjen et al. ${ }^{14}$ and Lo et al. ${ }^{26}$ studies showed that a greater chance of cognitive decline was found in patients with left-sided lesions.

In addition, the current study found that cognitive impairment patients had higher frequency of both (cortical-subcortical) strokes when compared with patients with normal cognition. In line with these results, Heiss WD et al. ${ }^{9}$ showed that vascular cognitive impairment is attributed to corticalsubcortical lesions as reaching a critical tissue loss or damage. While Cumming et al. ${ }^{27}$ and Flier et al. ${ }^{28}$ showed that cognitive impairment higher with cortical stroke.

On the other hand, Renjen et al. ${ }^{14}$ noted that patients with only subcortical lesion were more likely than those with a cortical lesion to have cognitive impairment.

Moreover, our study showed that patients with cognitive impairment had significantly larger stroke lesions when compared with patients with normal cognition. A study by Flier et al. ${ }^{27}$ showed that larger size of infarction increase the possibility of cognitive impairment. Also, Kalaria et al. ${ }^{13}$ and Cumming et $\mathrm{al}^{26}$ studies showed that in cognitively disabled patients, lesions appear to be larger than in nonimpaired patients, since larger strokes are more likely to affect cortical and other regions that support memory.
On the other hand in the study by Renjen et al. ${ }^{14}$ showed that the size or volume of the lesion had no correlation with cognitive impairment.

\section{CONCLUSION}

Cognitive impairment is prevalent in stroke patients. Risk factors included older age, left hemispheres strokes, hyperlipidemia, cortical and deep strokes and larger stroke lesions.

\section{REFERENCES}

1. Kapoor A, Lanctot KL, Bayley M, et al. Screening for Post-Stroke Depression and Cognitive Impairment at Baseline Predicts LongTerm Patient-Centered Outcomes After Stroke. $J$ Geriatr Psychiatry Neurol. 2019;32(1):40-8.

2. Puy L, Barbay $M$, Roussel $M$, et al. Neuroimaging determinants of poststroke cognitive performance: the GRECogVASC study. Stroke. 2018; 49(11):2666-73.

3. Jensen $M$ and Thomalla G. Causes and Secondary Prevention of Acute Ischemic Stroke in Adults. Hamostaseologie. 2020;40(1):22-30.

4. Saa JP, Tse T, Baum C, et al. Longitudinal evaluation of cognition after stroke-A systematic scoping review. PloS one. 2019 29;14(8):221735.

5. Rohde D, Gaynor E, Large M, et al. The impact of cognitive impairment on poststroke outcomes: A 5-year follow-up. J Geriatric Psyc Neurol. 2019; 32(5):275-81.

6. Nakling AE, Aarsland D, Næss $\mathrm{H}$, et al. Cognitive deficits in chronic stroke patients: neuropsychological assessment, depression, and self-reports. Dementia and Geriatric Cognitive Disorders Extra. 2017;7(2):283-96.

7. Kosgallana A, Cordato D, Chan DK, et al. Use of cognitive screening tools to detect cognitive impairment after an ischaemic stroke: a systematic review. SN Comprehensive Clinical Medicine. 2019;1(4):255-62.

8. Zhong W, Cruickshanks KJ, Huang GH, et al. Carotid atherosclerosis and cognitive function in midlife: the Beaver Dam Offspring Study. Atherosclerosis. 2011;219(1):330-3.

9. Heiss WD, Rosenberg GA, Thiel A, et al. Neuroimaging in vascular cognitive impairment: a state-of-the-art review. BMC Med. 2016; 14(1):174.

10. Mallery L, MacLeod T, Allen M, et al. Systematic review and meta-analysis of secondgeneration antidepressants for the treatment of older adults with depression: questionable benefit and considerations for frailty. $J$ BMC geriatrics. 2019;19(1):1-11.

11. Potocnik J, Stante KO and Rakusa M. The validity of the Montreal cognitive assessment (MoCA) for the screening of vascular cognitive impairment after ischemic stroke. Acta Neurol Belgica. 2020; 19:1-5.

12. Mellon L, Brewer L, Hall P, et al. Cognitive impairment six months after ischaemic stroke: a profile from the ASPIRE-S study. BMC Neurol. 2015;15(1):1-9. 
13. Kalaria RN, Akinyemi R and Ihara M. Stroke injury, cognitive impairment and vascular dementia. Biochim Biophys Acta. 2016;1862(5):915-25.

14. Reijmer YD, van den Berg E, Dekker JM, et al. Development of Vascular Risk Factors over 15 Years in Relation to Cognition: The $\mathrm{H}$ oorn Study. Journal of the American Geriatrics Society. 2012; 60(8):1426-33.

15. Wang A, Liu X, Chen G, et al. Association between carotid plaque and cognitive impairment in Chinese stroke population: The SOS-stroke study. Sci Rep. 2017;7(1):1-6.

16. Arntzen KA, Schirmer H, Johnsen SH, et al. Carotid artery plaque progression and cognitive decline: the T romsø Study 1994-2008. Eur J Neurol. 2012;19(10):1318-24.

17. Stramecki F, Kotowicz KD, Piotrowski P, et al Assessment of the association between cigarette smoking and cognitive performance in patients with schizophrenia-spectrum disorders: A casecontrol study. Frontiers in psychiatry. 2018; 9:642.

18. Rusanen M, Kivipelto M, Quesenberry CP Jr, et al. Heavy smoking in midlife and long-term risk of Alzheimer disease and vascular dementia. Arch Intern Med. 2011;171:333-9.

19. Sabia S, Elbaz A, Dugravot A, et al. Impact of Smoking on Cognitive Decline in Early Old Age: The Whitehall II Cohort Study. Arch Gen Psychiatry. 2012;69(6):627-35.

20. Renjen PN, Gauba C and Chaudhari D. Cognitive Impairment After Stroke. Cureus. 2015; 7(9):335

21. Akinyemi RO, Firbank M, Ogbole GI, et al. Medial temporal lobe atrophy, white matter hyperintensities and cognitive impairment among Nigerian African stroke survivors. BMC Res notes. 2015; 8(1):1-0.

22. Béjot Y, Aboa-Eboulé C, Durier J, Rouaud O, Jacquin A, Ponavoy E, et al. Prevalence of early dementia after first-ever stroke: a 24-year population-based study. Stroke. 2011 Mar; 42(3):607-12.

23. Chen PH, Cheng SJ, Lin HC, et al. Risk Factors for the Progression of Mild Cognitive Impairment in Different Types of Neurodegenerative Disorders. Behav Neurol. 2018 Jun;2018: 6929732.

24. Tu Q, Jin H, Ding B, et al. Reliability, Validity, and Optimal Cutoff Score of the Montreal Cognitive Assessment (Changsha Version) in Ischemic Cerebrovascular Disease Patients of Hunan Province, China. Dement Geriatr Cogn Disord Extra 2013;3:25-36.

25. Yang J, Wong A, Wang Z, et al. Risk factors for incident dementia after stroke and transient ischemic attack. Alzheimer's \& Dementia. 2015 Jan;11:16-23.

26. Lo JW, Crawford JD, Desmond DW, et al. Stroke and Cognition (STROKOG) Collaboration. Profile of and risk factors for poststroke cognitive impairment in diverse ethnoregional groups. Neurology. 2019; 93(24):2257-71.

27. Cumming TB, Marshall RS and Lazar RM. Stroke, cognitive deficits, and rehabilitation: still an incomplete picture. Int $J$ Stroke. 2013; 8(1):38-45.
28. van der Flier WM, Skoog I, Schneider JA, et al. Vascular cognitive impairment. Nat Rev Dis Primers. 2018; 4:18003. 